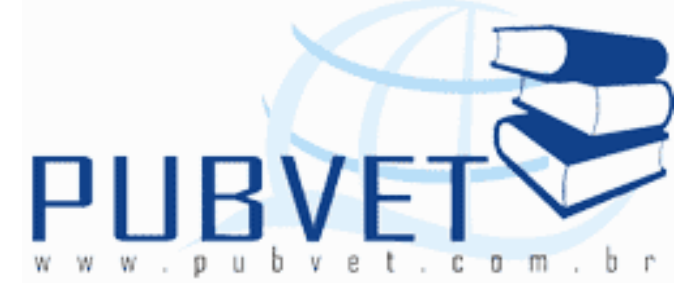

PUBVET, Publicações em Medicina Veterinária e Zootecnia.

\title{
Épulis periodontal em cães - aspectos macroscópico, histopatológico e citopatológico: quatro casos
}

Humberto Eustáquio Coelho ${ }^{1}$, Tatiane Furtado de Carvalho ${ }^{2}$, Letícia Oliveira Souza ${ }^{3}$, Ana Paula Porto Romão ${ }^{3}$ Flavia Maria Esteves Machado ${ }^{4}$, Claudio Henrique Gonçalves Barbosa ${ }^{5}$, Hélio Alberto ${ }^{6}$

${ }^{1}$ Professor de Patologia Animal - Universidade de Uberaba.

${ }^{2}$ Mestranda em Patologia Geral pela Universidade Federal de Minas Gerais.

${ }^{3}$ Alunas de graduação de Medicina Veterinária - Universidade de Uberaba.

${ }^{4}$ Professora de Epidemiologia e Zoonoses - Universidade de Uberaba.

${ }^{5}$ Residente em Patologia Animal do Hospital Veterinário de Uberaba.

${ }^{6}$ Farmacêutico e técnico do Laboratório de Anatomia Patológica do Hospital Veterinário de Uberaba

\section{Resumo}

Entre os locais mais acometidos pelas neoplasias em cães, estão os de cavidade oral e de faringe, destacando como mais frequentes o épulis e a papilomatose oral. As raças predispostas a esta patologia são o boxer e o Bull dog, sendo os animais do sexo masculino mais afetado. É uma patologia de caráter benigno localizados na gengiva próximo as dentes incisivos. No presente trabalho serão relatados quatro casos de épulis fibromatoso periodontal em cães machos, adultos, da raça boxer, diagnosticados entre 2010 a 2012, através da necropsia, no Laboratório de Patologia Animal do 
Hospital Veterinário de Uberaba. Clinicamente antes do óbito esses animais apresentavam sialorréia, dificuldade de se alimentar, perda de peso e halitose. Foi avaliado as características macroscópicas e celulares pela realização do histopatológico e citopatologia desta neoformação.

Palavras-chave: boxer; cães, épulis fibromatoso periodontal; neoplasia, parasitologia

\title{
Periodontal epulis in dogs - macroscopic, histopathology and cytopathology: four cases
}

\begin{abstract}
Among the most affected by neoplasia in dogs are the oral cavity and pharynx, highlighting the most frequent epulis and oral papillomatosis. The breeds are predisposed to this disease and the boxer, bull dog, while the male animals most affected. It is a benign disease of the gums located near the incisors. In the present work are reported four cases of periodontal fibromatous epulis in dogs boxer adult, male, diagnosed between 2010 to 2012, by autopsy in the Pathology Laboratory Animal Veterinary Hospital of Uberaba. Clinically, before death these animals showed salivation, difficulty eating, weight loss and halitosis. Was evaluated the macroscopic and cellular phones for carrying out the histopathology and cytopathology of bone formation.
\end{abstract}

Keywords: boxer; dogs, periodontal fibromatous epulis; tumor; parasitology

\section{INTRODUÇÃO}

Tanto a cavidade oral quanto a faringe estão entre os locais mais acometidos pelas neoplasias em cães (TODOROF e BRODEY, 1979). As neoplasias benignas mais comumente encontradas nos carnívoros domésticos são o épulis e a papilomatose oral (HARVEY e EMILY,1993).

O épulis é relativamente comuns nos cães, sendo raras nos gatos (WITHROW e VAIL, 2007), observando que raças predispostas a esta patologia 
são o boxer e o bull dog (FERRO et al., 2004), sendo que GIOSO (2007) acrescenta as raças pointer, weimaraner e cocker spaniel. A média de idade de apresentação para cães é entre 8 a 9 anos (WITHROW e VAIL, 2007). Animais do sexo masculino são os mais afetados (FERRO et al., 2004).

Esta patologia é uma neoformação da cavidade oral de caráter benigno que se origina do estroma do ligamento periodontal (OAKES et al., 1993; NORTH e BANKS, 2009) e normalmente estão localizadas na gengiva próximo aos dentes incisivos (THEILEN e MADEWELL, 1987).

Histologicamente o épulis é denso, possuindo um estroma bem vascularizado e não encapsulado. Observa-se a presença de células estreladas que possuem uma quantidade de colágeno fibrilar significativa, assemelhandose assim ao ligamento periodontal. Possui um núcleo irregular com características de mitose. Além disso, pode ocorrer uma pequena quantidade de eosinófilos (COLGIN et al., 2001).

O diagnóstico definitivo é baseado no resultado da avaliação histopatológica (MARRETTA, 1998). A técnica de citologia esfoliativa pode ser realizada para detectar carcinoma de células escamosas, além de detectar melanoma maligno e fibrossarcoma e tem sido provado ser um método simples, rápido e não traumático (CAVINA, 1964). Tal procedimento baseia-se na diminuição da coesão em células tumorais, que permitem a visualização mesmo que não seja de origem epitelial. Entratanto, alguns autores relatam que este método não substitui a biópsia (SANDLER et al., 1960).

O objetivo deste trabalho é relatar a ocorrência de quatro casos de Épulis periodontal em cães boxer, machos, adultos e avaliar as características macroscópicas e celulares a partir da realização do histopatológico e citopatologia desta neoformação.

\section{RELATO DE CASO}

Entre 2010 a 2012 foram diagnosticados 4 casos de épulis fibromatoso periodontal em cães machos, adultos, da raça boxer, necropsiados no 
Laboratório de Patologia Animal do Hospital Veterinário de Uberaba. Clinicamente antes do óbito esses animais apresentavam sialorréia, dificuldade de se alimentar, perda de peso e halitose.

Macroscopicamente foi possível observar um crescimento tumoral firme e sólido e de aspecto pedunculado, crescendo ao redor da gengiva (Fig. 1). Foram coletadas amostras destas neoformações para a realização do histopatológico e realizou-se no momento um "imprint" como método citopatológico. Na citopatologia observou-se células com citoplasma azul claro abundante, núcleo pequeno e hipercromático.

No exame histopatológico observou-se células com núcleo irregular, estroma denso, membrana celular e membrana nuclear irregulares e vacúolo pequeno e com uma fina porção de cromatina. Identificou-se significativa característica mitótica e a presença de eosinófilos e células estreladas, além de intensa vascularização local (Fig. 2, 3).

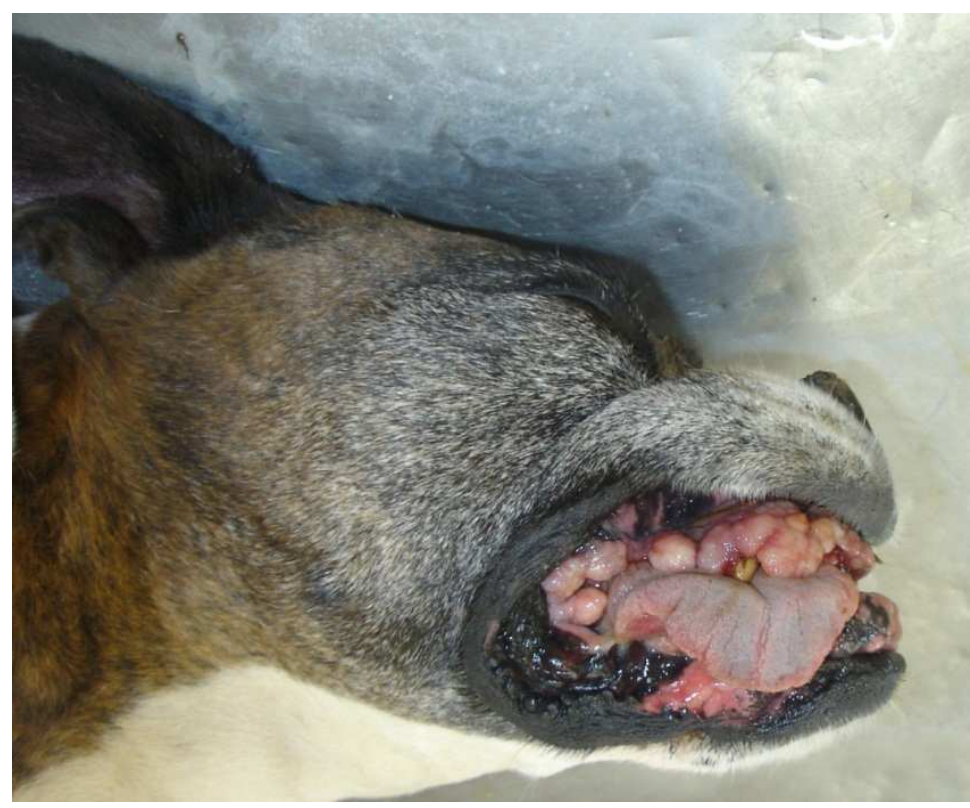

Figura 1: Épulis periodontal em um cão. Aspecto macroscópico do crescimento tumoral ao redor da gengiva, possuindo característica firme, sólida e pedunculada. 


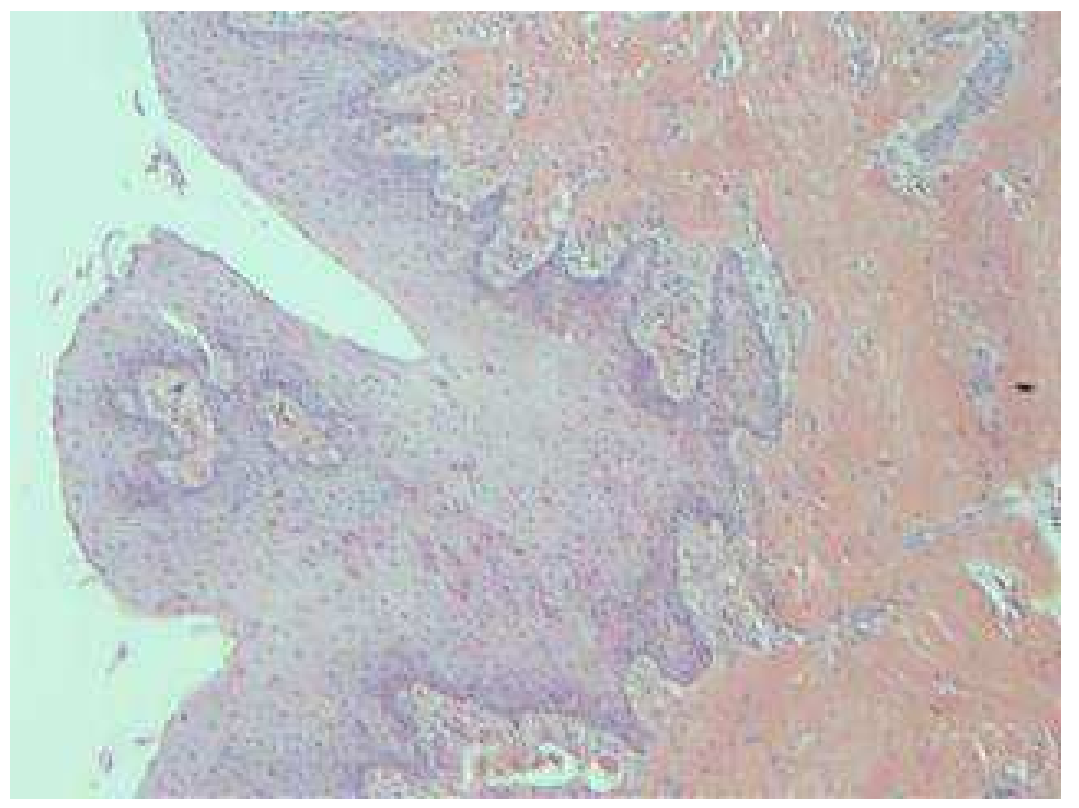

Figura 2 - Épulis periodontal em um cão. Epitélio recoberto com células escamosas, e mostra variações no comprimento das criptas epiteliais. Coloração HE. Aumento de 4x.

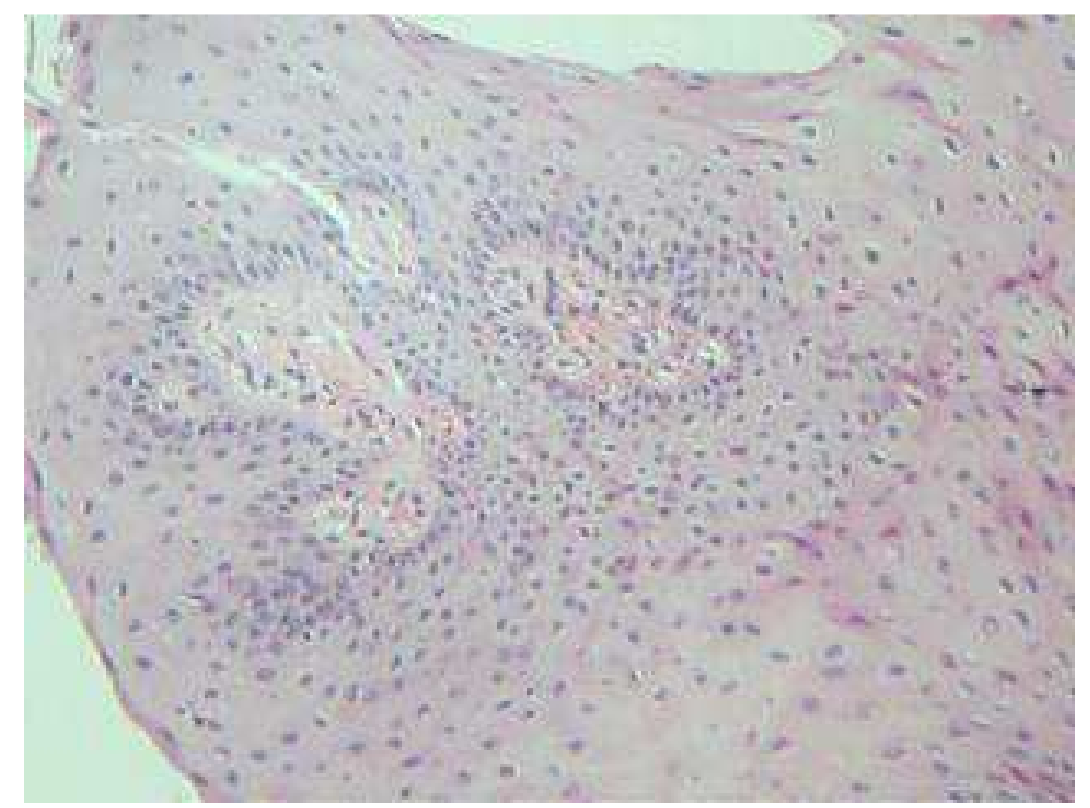

Figura 3 - Épulis periodontal em um cão. Composta por tecido conjuntivo apresentando células alongadas com núcleo hipercromático alongado e citoplasma eosinofílico filamentar, presença de estroma de ligamento periodontal com ninhos de matriz colágena e ausência de figuras mitóticas. Coloração HE. Aumento de 10x. 


\section{DISCUSSÃO}

A incidencia maior de épulis periodontal fibromatoso ocorreu na região molar e insivivos, de acordo com MOULTON (1990) o épulis solitário pode estar associado com todos os dentes, mas eles provavelmente ocorrem com mais freqüência na região molar, especialmente ao redor dos dentes carniceiro, em seguida, nas regiões dos dentes pré-molares e caninos, e menos comumente em torno dos incisivos. A superfície pode tornar-se ulcerada e infectada por causa dos danos oclusais.

Esta neoformação pode ser classificada como acantomatosa, ossificante ou fibromatosa (OAKES et al., 1993; THEILEN e MADEWELL, 1987). A forma fibromatosa é a mais frequente e não possui comportamento invasivo, podendo englobar o dente (ROZA, 2004). O estroma celular denso e o colágeno assemelham-se à membrana periodontal. Normalmente está presente pequena quantidade de osteóide, dentina e cemento, além de restos de epitélio laminar dental (SAN-ROMÁN, 1999).

Macroscopicamente foi observado um crescimento tumoral firme e sólido e de aspecto pedunculado, crescendo ao redor da gengiva, de acordo com ROZA (2004) esta neoformação apresenta superfície lisa e pode-se estender pelo sulco gengival, e envolver os dentes. COLGIN et al. (2001) acrescenta que sua cor varia de cinza a vermelho, podendo possuir poucos milímetros de largura até dimensões que chegam a ultrapassar ao dente associado

Os achados microscópicos condizem com MOULTON (1990), que descrevem a lesão é composta de forma compacta, grossas fibras, colágeno moderadamente, numerosas células estreladas e uma dispersão regular de pequenos vasos sanguíneos que, perto da superfície epitelial, pode mostrar um padrão de tecido de granulação. Infiltração de células plasmáticas e linfócitos é encontrada frequentemente relacionados à ulceração do epitélio de superfície. O epitélio não está espessado. As células periféricas são colunares e em paliçada; as células centrais das massas maiores são os queratinócitos típico com pontes intercelulares, mas é encontrada queratina "pérolas". Cistos 
ocasionais revestidos por epitélio escamoso estratificado estão presentes. Figuras de mitose são raras tanto tecido epitelial e fibroso. Nas áreas mais profundas da lesão, há osteóide e ósseo maduro lamelar associada com célulamatriz rica formadoras de osso.

O épulis periodontal tem geralmente crescimento hemisférico nodular que se projetam a partir da gengiva, mas crescem lentamente e pode infiltrar o osso, mas causam proliferação óssea pouca ou nenhuma (MOULTON, 1990).

$\mathrm{Na}$ citopatologia os epúlis são compostos por epitélio escamoso e tecido fibroso, aspirados destes tumores sao compostos por quantidade variável de células escamosas maduras e pequenas células espinais ocasionais. A porçao fibrosa do epulis é não esfoliativa ou minimamente esfoliativa, o que faz com que muitos aspirados sejam quase acelulares e nao diagnósticos (COWELL et al., 2009).

\section{REFERENCIAS BIBLIOGRÁFICAS}

CAVINA, C. La citologia esfoliativa nella diagnosi dei tumori del cavo orale. ArC Stomatol, v. 5, p 485-507, 1964.

COLGIN, L. M. A.; SCHULMAN, F. Y., DUBIELZIG, R. R. Multiple epulides in 13 cats. Veterinary Pathology, Washington, v. 38, n. 2, p. 227-229, 2001.

COWELL. R. L. ; TYLER, R. D.; MEIINKOTH, J. H.; DENICOLA. Diagnóstico citológico e hematologia de cães e gatos. 3ed .São Paulo:Medvet,.Cap.4,p 68-77. 2009

FERRO, D.G.; LOPES, F.M.; VENTURINI, M.A.F.A.; CORREA, H.L.; GIOSO, M.A. Prevalência de neoplasias da cavidade oral de cães atendidos no Centro Odontológico Veterinário - Odontovet ${ }^{\circledR}-S P$ - entre 1994 e 2003. Arq. ciên. vet. zool. UNIPAR, 7(2): p. 123-128, 2004.

GIOSO, M.A. Odontologia Veterinária para o clínico de pequenos animais. Ed.2 São Paulo: Manole, p. 91, 2007.

HARVEY, C.E.; EMILY, P.P. Oral neoplasms. In: Smal animal dentistry, St. Louis, Mosby, 1993.

MARRETTA, S. M. Maxillofacial surgery. The Veterinary Clinics of North America Small Animal Practice, v. 28, n. 5 , p. 1285-1296, 1998.

MOULTON, J.E. Tumors in domestic animals. 3.ed. London : University of California,672p, 1990. 
NORTH, Susan; BANKS, Tania. Introductio to small animal oncology: Tumours of head and neck. Louis, Missouri: Saunders Elsevier, 2009.

OAKES, M.G.; LEWIS, D.D.; HEDLUND, C.S.; HOSGOOD, G. Canine oral neoplasia. The Compendium, v.15, n.1, p.15-31, 1993.

ROZA, M.R. Odontologia em Pequenos Animais. Rio de Janeiro: L.F. Livros, p. 304, 2004.

SANDLER, H.C., STAHL, S., CAHN,L.R. Exfoliative cytology for detetion of early mouth cancer. Oral Surg,Oral Med, Oral Path, v. 13, p. 994-1009, 1960.

SAN-ROMÁN, F. Atlas de odontologia de Pequenos Animais. São Paulo: Manole, p 144157, 1999.

THEILEN, G. H.; MADEWELL, B. R.: Tumors of the digestive tract. Veterinary Cancer Medicine. Philadelphia: Lea eFebiger, p. 499-534, 1987.

TODOROFF, R.J.; BRODEY, R.S. Oral and pharyngeal neoplasia in the dog: a retrospective survey of $\mathbf{3 6 1}$ cases. J Am Vet Med Assoc, v.175, n.15, p.567-571, 1979.

WITHROW, S.J.; VAIL, D.M. Small animal clinical oncology. Missouri: Saunders- Elsevier, p. 457-467, 2007. 